\title{
Low-dose sertraline improves receptive language in children with fragile $x$ syndrome when eye tracking methodology is used to measure treatment outcome
}

\begin{abstract}
Background: Language impairments constitute a significant part of the phenotype of individuals with fragile X syndrome (FXS)-the most common form of inherited intellectual disability. It has been hypothesized that a selective serotonin reuptake inhibitor (SSRI) may improve language development in children with FXS by regulating serotonin levels. However, due to social anxiety and withdrawal symptoms in children with FXS, it is difficult to collect reliable measurements of language abilities in these children using standardized, in-person assessments. The present study examined:

a. The utility of using eye tracking methodology to assess language abilities in children with FXS.

b. The effect of sertraline on language improvement accessed via an eye tracking task in a randomized, double-blind, placebo-controlled trial in children with FXS.

Methods:The study had two parts. First, a passive-viewing eye tracking task (PVET) was used to examine receptive language in children with FXS. We then compared the performance on the PVET to parent report of child's language abilities. Second, the PVET was used to assess receptive language at baseline and at 6-months follow-up, in children with FXS enrolled in a randomized, double-blind, placebo-controlled trial of sertraline lasting 6 months. We also examined language skills, pre- and post-treatment, with two standardized behavioral assessments: the receptive language subscale of the Mullen Scales of Early Learning (MSEL) and the Preschool Language Scale (PLS).

Results: Performance on the PVET was consistent with parent report of child's language ability. Further, children who received sertraline, compared to children in the placebo group, performed better on the PVET post-treatment compared to baseline while no significant improvement in receptive language was observed when assessed using the MSEL or the PLS scores.

Conclusion:We provide evidence for using eye tracking methodology as a measure of receptive language in children with FXS. Further, we provide preliminary evidence of sertraline as an effective treatment in improving receptive language in young children with FXS using eye tracking. Future investigations of treatment in children with FXS should explore eye tracking methodology in conjunction with standardized assessments as outcome measures.
\end{abstract}

Trial registration:Study information can be found on clinicaltrials.gov. The trial registration identifier is NCT01474746, and the registration date is November 10, 2011.

Keywords: fragile x syndrome, sertraline, ssri, autism, eye tracking, receptive language
Volume 7 Issue 6 - 2017

\author{
Kathryn HYoo, ${ }^{1,2}$ Jessica L Burris, ${ }^{2}$ Kimberly \\ N Gaul, ${ }^{3}$ Randi J Hagerman, ${ }^{1,4}$ Susan M \\ Rivera ${ }^{1,2}$ \\ 'Medical Investigation of Neurodevelopmental Disorders \\ (MIND) Institute, University of California, USA \\ 2Department of Psychology, University of California, USA \\ ${ }^{3}$ Department of Social Welfare, University of California, USA \\ ${ }^{4}$ Department of Pediatrics, University of California Davis \\ Medical Center, USA
}

Correspondence: Susan M Rivera, Department of Psychology, University of California, Davis, 95618 and the MIND Institute, USA, Email srivera@ucdavis.edu

Received: June 08, 2017 | Published: June 26, 2017
Abbreviations: FXS, fragile $\mathrm{x}$ syndrome; PVET, passiveviewing eye tracking task; MSEL, mullen scales of early learning; PLS, preschool language scale; FMR1, fragile x mental retardation 1; FMRP, fmr1 protein; SSRI, selective serotonin reuptake inhibitor; ASD, autism spectrum disorder; CA, chronological age; MA, mental age; CDI, macarthur-bates communicative development inventory

\section{Background}

Fragile X syndrome (FXS) is a X-linked genetic disorder caused by a trinucleotide CGG repeat expansion of over 200 repetitions on the fragile X mental retardation 1 (FMR1) gene at Xq27.3 ${ }^{1}$ and is the most common form of inherited intellectual disability. ${ }^{2}$ The full FXS mutation typically causes FMR1 methylation, resulting in significantly reduced or absent levels of the FMR1 protein (FMRP)-a selective, inhibitory, mRNA-binding protein that regulates the translation of mRNAs into their respective proteins. This deficit in FMRP results in intellectual, cognitive, and behavioral impairments including anxiety, attention-deficit hyperactivity disorder, aggression, self-injurious behaviors, deficits in sensory processing, and language impairments. ${ }^{3-8}$

Although FMRP is expressed throughout the body, this protein plays a critical role in the development of neuronal soma and dendrites as FMRP regulates most of the proteins important for synaptic plasticity. ${ }^{9}{ }^{10}$ Specifically, FMRP negatively regulates the translation of RNA species into cytoskeleton proteins, $\gamma$-aminobutyric acid receptor subunits, and metabotropic glutamate receptors (mGluRs) at the 
synapse. ${ }^{10-12}$ In FXS the lack of FMRP, and typically up-regulation of many proteins whose translation is inhibited by FMRP results in long, spindly, immature dendritic spines and negatively affects synaptic plasticity, synaptic transmission, and learning and memory. ${ }^{13}$ Due to the upregulation of protein synthesis and altered synaptic function caused by deficits in FMRP levels, targeted treatments for FXS seek to reverse these neurophysiological changes.

One proposed treatment for ameliorating symptoms of FXS is the use of selective serotonin reuptake inhibitors (SSRIs). Existing research shows that children with autism spectrum disorder (ASD) have lowered levels of serotonin production in the CNS in the first 5 years of life compared to neurotypically developing children. ${ }^{14,15}$ Further, treatment in early childhood may have cascading effects into later years of life due to rapid brain development during this early period. ${ }^{16,17}$ Although treatment trials assessing the efficacy of SSRIs in children with disabilities are few, existing studies in $\mathrm{FXS}^{18,19}$ and ASD, which has a high co-morbidity with FXS, ${ }^{20}$ suggest that SSRIs may be beneficial in reducing symptoms of anxiety, irritability, and transition-induced behavioral deterioration, and may improve expressive and receptive language development, fine motor skills, and social participation.

Significantly diminished vocabulary, as compared to typically developing children, is a hallmark feature of FXS. ${ }^{21-23}$ Further, FXS is associated with impairments and delays in various cognitive domains relevant for language acquisition such as sequential processing, ${ }^{24}$ working memory, ${ }^{25}$ and attention. ${ }^{26}$ Not only do these cognitive challenges impact language abilities, but individuals with FXS exhibit relatively higher rates of challenging behaviors and psychopathology that may likely disturb the social interactions that promote language development. These include hyperactivity, ${ }^{27}$ avoidance issues as a result of neuroendocrine dysfunction, ${ }^{28}$ and social anxiety. ${ }^{29,30}$ The present study examined the efficacy of sertraline on the development of language in children with FXS. Sertraline is approved by the FDA as a treatment for obsessive-compulsive disorder in children aged 6-17years, and for major depressive disorder, panic disorder, posttraumatic stress disorder, premenstrual dysphoric disorder, and social anxiety in adults. To date, few studies have examined the effect of low-dose sertraline on language development in children with FXS. In one study with 12-50month-olds with FXS, Winarni and colleagues found improvement in both expressive and receptive language development in the group treated with low-dose sertraline. ${ }^{19}$ However, the results from this study were from retrospective observation data obtained from a chart review of medical records, which could introduce clinical bias in addition to other potential confounding factors associated with treatment that were not available in the medical records.

In a controlled trial of low-dose sertraline in young children with FXS aged 2-6years old, Greiss-Hess and colleagues found significant improvements in motor and visual perceptual abilities and social participation in the group treated with sertraline compared to the placebo group, but they did not find significant improvements in language development for the group as a whole. ${ }^{18}$ However, post hoc assessment for those with FXS and ASD found significant improvement in expressive language compared to placebo. GreissHess and colleagues used clinical assessments, which required interactions with clinicians as well as responses from the child with FXS. It is possible that Greiss-Hess and colleagues did not find significant language improvements for the overall group because they utilized assessments requiring child-to-clinician interactions. Given the prominent social anxiety and withdrawal symptoms often seen in children with $\mathrm{FXS},{ }^{8}$ collecting accurate measurements of language abilities using standardized, in-person assessments may hamper language assessment in these individuals and cause an underestimation of skills.

In the present study, we present a novel, passive-viewing eye tracking task (PVET) as an alternative measure to assess language improvement in children with FXS over the course of low-dose sertraline treatment. The PVET was adapted from Fernald and colleagues' "looking-while-listening" task, which measures the time course of children's gaze patterns in response to speech. ${ }^{31}$ This methodology has low task demands and does not require verbal or overt motor responses such as a point from the child, which is ideal for children with FXS. Indeed, eye tracking methodology has been used successfully to examine speech comprehension in children with ASD. ${ }^{32}$ The current study had two aims. First, we examined the utility of the PVET to evaluate receptive language skills in young children with FXS. Second, we used the PVET as an exploratory outcome measure of language improvement in a randomized double-blind placebo-controlled trial of low-dose sertraline in children with FXS.

\section{Methods}

\section{Participants}

The sample to examine the utility of the PVET comprised 68 children diagnosed with FXS (55 male) with mean chronological age (CA) of 37.81 months ( $\mathrm{SD}=15.46 \mathrm{months} ; 12$ to 72 months). A subset of the sample for the PVET validity analysis were enrolled in a placebocontrolled study of sertraline. The sample for the placebo-controlled study of sertraline comprised 22 children, 24 to 60 months, diagnosed with FXS (21 male), randomly assigned to either placebo $(n=12)$ or sertraline $(\mathrm{n}=9)$ and comprised a subset of the sample reported in. ${ }^{18}$ These children were evaluated at two time points: baseline and 6-months follow-up. For the placebo group at baseline, the mean CA was 43.14 months ( $\mathrm{SD}=14.52 \mathrm{months} ; 2$ to 5years) and at the followup, the mean CA was 51.54 months ( $\mathrm{SD}=12.92$ months; 2 to 5 years). For the sertraline group at baseline, the mean CA was 41.54 months ( $\mathrm{SD}=12.34$ months; 2 to 5years) and at the follow-up, the mean CA was 47.79 months ( $\mathrm{SD}=12.27$ months; 2 to 5years).

Participants were clinically evaluated at the UC Davis MIND Institute. Participants were English speaking and willing to travel and participate in the current trial. Participants with central nervous system disease other than FXS were excluded from the study and participants with and without ASD were included in the study. Participants 2 to 3 years of age received sertraline liquid or placebo liquid in a dose of $2.5 \mathrm{mg} /$ day $(0.125 \mathrm{~mL})$ and participants older than 3years received a dose of $5.0 \mathrm{mg} /$ day $(0.25 \mathrm{~mL})$. Assessments were completed in the clinic at baseline and at a 6-month follow-up visit. In the first month, telephone calls were made weekly to monitor side effects with subsequent calls made monthly, and the 3-month follow-up included a review of behavior and side effects ${ }^{18}$ for details and profiles of the participants).

Cognitive level was assessed using the Mullen Scales of Early Learning, ${ }^{33}$ which is a standardized assessment used for children between 3 months and 60months of age. The MSEL consists of five subscales: gross motor, fine motor, receptive language, expressive language, and visual reception. The mental age (MA) of each participant was calculated by averaging the age equivalencies across four different domains (excluding gross motor) and converting this average to MA in months and days. We excluded the gross motor subscale from the MA calculation because, given our wide age range, the gross motor scores 
are less valid in children above the age of 33 months. ${ }^{33}$ In addition, the Preschool Language Scale (PLS) was administered to assess language skills. The PLS is consisted of the auditory comprehension scale (AC) and the expressive communication scale (EC) for children from birth through 7years and 11 months of age. ${ }^{34}$ The mental ages for the PVET (Aim 1) ranged from 5.23months to 71.18 months $(\mathrm{M}=21.82$ months; $\mathrm{SD}=11.19$ months). For the placebo-controlled sertraline study (Aim 2 ), MA at baseline for the placebo group ranged from 10 months to 35.21 months $(\mathrm{M}=21.17$ months; $\mathrm{SD}=7.58$ months $)$ and at follow-up, the MA ranged from 15.21 months to 39 months $(\mathrm{M}=25.58$ months; $\mathrm{SD}=7.89$ months). The MA at baseline for the sertraline group ranged from 6.21 months to 32.15 months $(\mathrm{M}=20.68$ months; $\mathrm{SD}=$ 8.11 months) and at follow-up, the MA ranged from 7.21months to 45.08months ( $M=25.46$ months; $S D=11.09$ months). See table 1 for CAs and MAs for each group at baseline and at follow-up.

Table I Age distribution (in months) of participants in the sertraline study

\begin{tabular}{lllll}
\hline & $\begin{array}{l}\text { Baseline } \\
\text { CA }\end{array}$ & MA & Follow-Up \\
& CA & MA \\
\hline Placebo & $43.14(14.52)$ & $21.17(7.58)$ & $51.54($ I2.92) & $25.58(7.89)$ \\
\hline Sertraline & $41.54($ I2.34) & $20.68(8.11)$ & $47.79(12.27)$ & $25.46(11.09)$ \\
\hline
\end{tabular}

Mean chronological age (CA) and mean mental age (MA) in months of participants in the placebo-controlled sertraline study at baseline and at follow-up. Standard deviations are in parentheses

\section{Apparatus}

Stimuli were presented on a 17-inch Tobii 1750 LCD binocular eye tracker (Tobii Technology, Sweden, 1280 x 1024 pixels resolution) to record children's fixations during the task. Eye tracking data were collected at a sample rate of $50 \mathrm{~Hz}$. The average accuracy of the recorded eye coordinates was about $0.5 \mathrm{o}$, which is approximately $0.5 \mathrm{~cm}$ at a viewing distance of $60 \mathrm{~cm}$. The average accuracy in timing is $25-35 \mathrm{~ms}$. Drifts are compensated with an average error of $0.5 \mathrm{o}$. When one eye could not be measured, data from the other eye were used to determine the gaze coordinates. The recovery time to full tracking ability after an offset was about $100 \mathrm{~ms}$. Fixations were defined using the Tobii fixation filter, such that maximum angle between fixations was $0.5 \mathrm{o}$ and fixations had to be longer than $60 \mathrm{~ms}$. A standard fivepoint Tobii calibration was attempted for all participants. Recalibration occurred if any single point was missed. Our goal was to only begin the task once all 5 points had a successful calibration; however, given the limited attention span and behavioral challenges that are present in children with FXS, the minimum requirement to proceed with the task was a successful calibration of the center point for each eye.

\section{Task details}

Cognitive level was assessed using the Mullen Scales of Early Learning, ${ }^{33}$ which is a standardized assessment used for children between 3 months and 60 months of age. The MSEL consists of five subscales: gross motor, fine motor, receptive language, expressive language, and visual reception. The mental age (MA) of each participant was calculated by averaging the age equivalencies across four different domains (excluding gross motor) and converting this average to MA in months and days. We excluded the gross motor subscale from the MA calculation because, given our wide age range, the gross motor scores are less valid in children above the age of 33 months. ${ }^{33}$ In addition, the Preschool Language Scale (PLS) was administered to assess language skills. The PLS is consisted of the auditory comprehension scale (AC) and the expressive communication scale (EC) for children from birth through 7years and 11 months of age. ${ }^{34}$ The mental ages for the PVET (Aim 1) ranged from 5.23months to 71.18 months $(\mathrm{M}=21.82 \mathrm{months}$;
$\mathrm{SD}=11.19$ months). For the placebo-controlled sertraline study (Aim 2), MA at baseline for the placebo group ranged from 10months to 35.21 months $(\mathrm{M}=21.17$ months; $\mathrm{SD}=7.58$ months $)$ and at follow-up, the MA ranged from 15.21 months to 39 months $(\mathrm{M}=25.58$ months; $\mathrm{SD}=7.89$ months). The $\mathrm{MA}$ at baseline for the sertraline group ranged from 6.21 months to 32.15 months $(\mathrm{M}=20.68$ months; $\mathrm{SD}=$ 8.11 months) and at follow-up, the MA ranged from 7.21months to 45.08months ( $\mathrm{M}=25.46$ months; $\mathrm{SD}=11.09$ months). See table 1 for CAs and MAs for each group at baseline and at follow-up.

The task consisted of 30 objects selected from the MacArthurBates Communicative Development Inventory (CDI) questionnaire of commonly known words by age. ${ }^{34}$ Images of selected words were taken from image sets created from the original Snodgrass and Vanderwert's object database that contained texture and color information. ${ }^{35,36}$ In the scenario that a selected word did not have a corresponding picture, an additional word was selected from the same age category of words.

Images were paired side by side (two images per trial) presented on a white background at a viewing distance of $60 \mathrm{~cm}$ with a visual angle of approximately $6.78^{\circ}$. Each image was used once as a target word and once as a distractor word resulting in 30 total trials in the task. Following Fernald and colleagues ${ }^{31}$ guidelines, animate items (e.g., duck) were paired with animate items (e.g., cat), and inanimate items (e.g., book) were paired with inanimate items (e.g., chair). Aside from these guidelines, pairings were randomly selected. Each image pair was presented for a total of six seconds in a fixed random order to all participants with a 1-second fixation cross that was presented between image pairs (Figure 1).

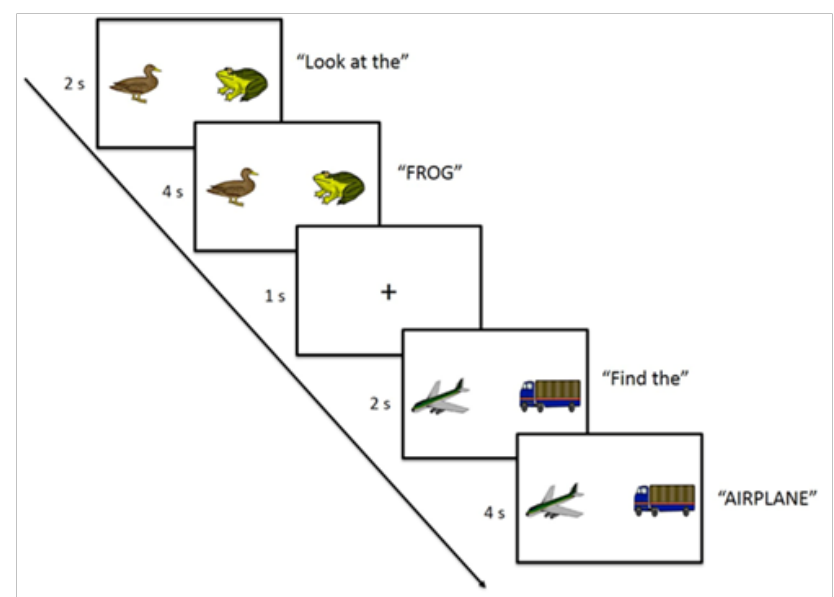

Figure I Sequence of events in the passive-viewing eye tracking task (PVET). The sequence illustrated here consists of two trials.

Audio clips were recorded with a female voice using a positive tone and affect for all target words and three different carrier phrases. The three different carrier phrases used were: "Where's the", "Find the", and "Look at the." Target words were recorded separately from the carrier phrases to control the timing of the target word presentation in each trial. The sound clips were edited together in Audacity ${ }^{\circledR}$ (www.audacityteam.org) to fluidly present a randomly selected carrier phrase with each of the 30 target words (e.g., "Where's the dog?", "Look at the ball"). These sound clips were recorded and combined in this way so that the onset of the target word occurred precisely two seconds into the visual presentation of the images.

A single trial consisted of the pair of objects appearing on the screen while the carrier phrase was presented and then exactly two seconds into the trial, the target label would begin. The images were 
shown prior to the label to allow for visual detection of both objects prior to the label being heard. The image then remained on the screen for four additional seconds to allow for visual reaction of the participant in response to the verbal instructions that they had heard. Each trial therefore lasted for a total of six seconds (Figure 2 for the schematic time line of a trial).

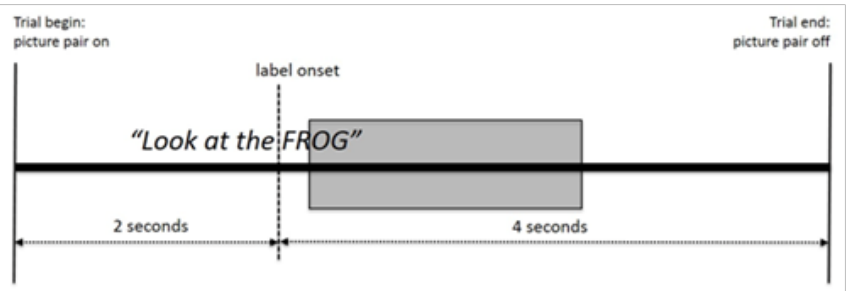

Figure 2 Schematic time line for a trial. The shaded area represents the 300 $\mathrm{ms}$ to $1800 \mathrm{~ms}$ segmentation window from label onset for calculating the visual preference score.

Prior to completion of the eye tracking protocol, parents completed a parent report language inventory for their child that asked the parents what words the child understands and says (Appendix A). The inventory consisted of two columns ( 30 words in each column) representing two versions of the task (older versus younger). This inventory was reviewed by a researcher prior to calibration to best choose which version of the task to present to the child. If children were marked as understanding less than $50 \%$ of the words on the younger version of the list, they were shown the younger version of the task (regardless of chronological age of the participant). Given that language deficits are a hallmark of FXS, ${ }^{3-37}$ we felt this was the best way to tailor presentation to each participant. We therefore had a parent report of whether the children were familiar with each word as well as the child's task performance on each presented word.

\section{Analysis}

The task was analyzed using Tobii Studio analysis software to calculate total looking time to each object on the screen. Statistical analyses were performed using SPSS (version 23). Scenes were created separately to capture the first two seconds of the trials (prelabel) and the last four seconds (post-label). This allowed for analyses of looking prior to the labeling of the target object (for baseline visual preference, and to ensure that both objects had been viewed) as well as visual reaction time to the auditory cue. Areas of interests (AOIs) were created to encompass $15 \%$ of the screen each around both the target and distractor objects. The primary measure of performance on each trial was the total fixation duration from $300 \mathrm{~ms}$ to $1800 \mathrm{~ms}$ after the onset of the target word (i.e., label), from which we calculated an overall visual preference score. The $300 \mathrm{~ms}$ to $1800 \mathrm{~ms}$ segmentation window was based on previous methods and findings. ${ }^{31-38}$ The visual preference score, or the proportion of looking time to target on each trial, was calculated as the total fixation duration to target object divided by the sum of the total fixation to target object and to distractor object.

\section{Results}

\section{Utility of the passive-viewing eye tracking task (PVET)}

We first examined the relations between the child's performance on the PVET (visual preferences score for target objects) and parentreport of the child's knowledge of the target object labels. We ran a one-sample t-test to examine whether the proportion of looking time for items the parent reported that the child knows, i.e., target objects, was significantly above chance $(.50)$. We found that proportion of looking time for items the parent reported as known to the child was significantly above chance for the target objects $(t(60)=2.703, p=$ .009 ; Figure 3). Next, we ran a one-sample t-test to assess whether the proportion of looking time for objects that the parent reported the child does not know was significantly above chance. Children did not look significantly longer at objects reported by the parent as unknown to the child $(t(53)=-.176, p=.861$; Figure 3$)$ i. Mean proportion of looking time for known objects versus unknown objects was 0.5531 $(\mathrm{SD}=.152)$ and $0.496(\mathrm{SD}=.183)$ respectively.

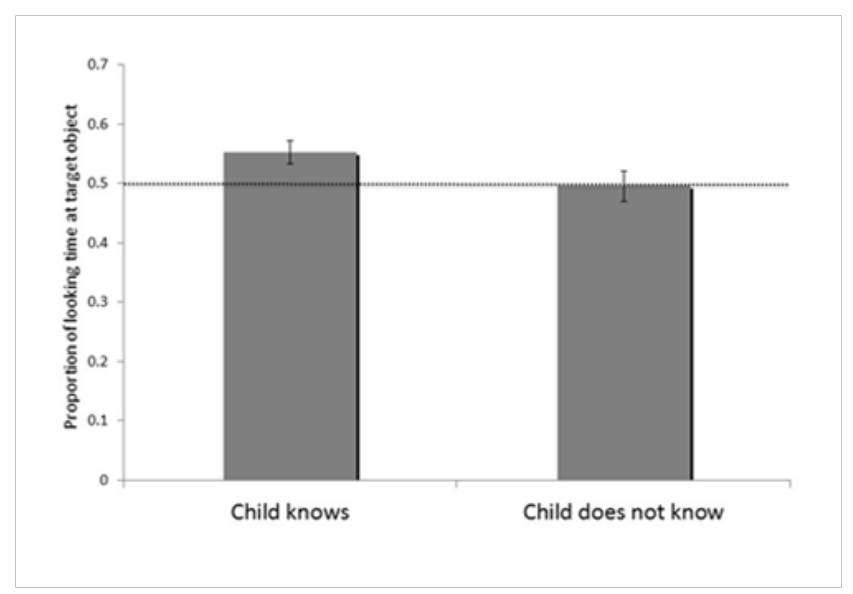

Figure 3 Parent report of child's word knowledge and performance on PVET.

Next, we assessed whether children's performance on the PVET was related to existing standardized assessments of receptive language. We assessed children's receptive language using the receptive language (RL) subscale of the Mullen Scales of Early Learning (MSEL) and the auditory comprehension (AC) subscale of the Preschool Language Scale (PLS). We ran a partial correlation and found a significant positive relation between looking time to target object and MSEL RL score $(\mathrm{r}(28)=.523, p=.003)$. Further, we found a significant relation between looking time to target and PLS AC score $(\mathrm{r}(28)=.565, p=.001)$. Scatterplots of these relations are provided in Figure 4.

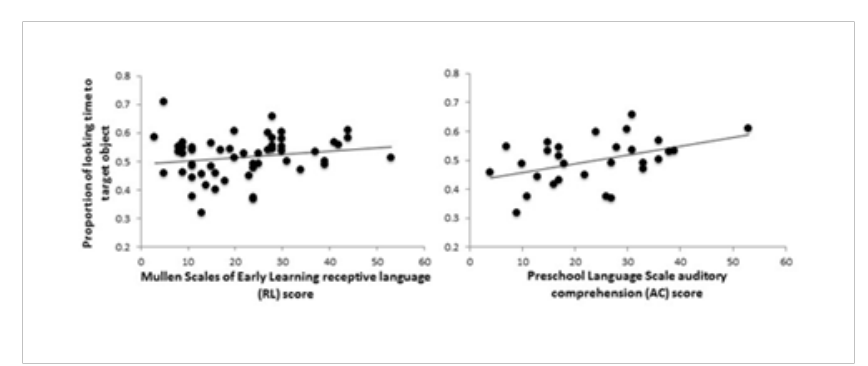

Figure 4 Relation between looking time to target and the age-equivalent score of the receptive scale of the Mullen Scales of Early Learning (left panel). Relation between looking time to target and the age-equivalent score of the auditory comprehension scale of the Preschool Language Scale (right panel).

\section{The effect of sertraline on PVET performance and assessment scores}

Using the PVET as an exploratory outcome measure, we examined whether sertraline influenced language improvement in children with FXS in a placebo-controlled trial. We implemented the PVET at baseline (pre) and at the 6-month follow-up (post) for both the placebo and sertraline groups. To examine whether sertraline influenced children's performance on the passive viewing task, we ran 
a repeated-measures analysis of variance (RMANOVA) with Group (placebo, sertraline) as a between-subjects factor and Phase (baseline, post) as the within- subjects factor. We found a main effect of Phase Follow up analyses revealed that for the sertraline group, there was a significant improvement in looking time on the PVET from baseline $(\mathrm{M}=.451 ; \mathrm{SD}=.070)$ to post $(\mathrm{M}=.538 ; \mathrm{SD}=.095 ;$ Figure 5$)$. The placebo group, however, did not show this improvement on the PVET task from baseline to post $\left(\mathrm{M}_{\text {baseline }}=.510, \mathrm{SD}_{\text {baseline }}=.056 ; \mathrm{M}_{\text {post }}=.506\right.$, $\mathrm{SD}_{\text {post }}=.080$ ).

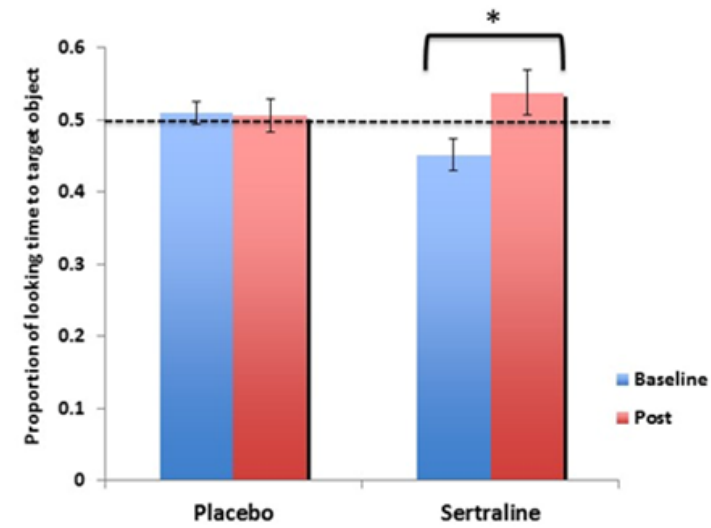

Figure $\mathbf{5}$ The effect of low-dose sertraline on looking time to target on the passive-viewing eye tracking task (PVET).

In addition, we examined whether the MSEL and the PLS reflected the efficacy of sertraline that was observed with improvement in the PVET from pre- to post-administration of sertraline. We ran a RMANOVA with Group (placebo and sertraline) as a betweensubjects factor and Phase (baseline and post) and Assessment (MSEL RL subscale and PLS AC subscale) as within-subjects factors. We found no significant interactions (Assessment $\mathrm{x}$ Group: $p=.92$; Phase x Assessment: $p=.87$; Phase $\mathrm{x}$ Assessment $\mathrm{x}$ Group: $p=.23$ ) in the assessment scores from pre to post after administration of sertraline or placebo suggesting that while the PVET was able to measure improvement in language comprehension, this improvement was not detected with the behavioral assessments MSEL and PLS.

\section{Discussion}

The current study first examined the utility of using eye tracking methodology to assess language abilities in young children with FXS. Measuring language skills, particularly receptive language in children with neurodevelopmental disorders, poses a challenge given that many standardized measures of language development including language comprehension are not suitable for children with neurodevelopmental disorders. In a study with children with ASD, it was reported that almost thirty percent of children performed at floor level with standardized assessments. This is potentially due to the fact that response demands (verbal or motor) are often required by traditional language assessments, leading to the underestimation of children's receptive language skills. ${ }^{40}$

The PVET used in the current study was adapted from the lookingwhile-listening methodology pioneered by Fernald and colleagues. ${ }^{31}$ and is technically "simple" with regards to the presentation of the stimuli and task demands. Therefore, this task was ideal to use with FXS populations given that overt verbal and motor responses were not required. Because individuals with FXS face both fine and gross motor problems and social anxiety ${ }^{41,42}$ this passive-viewing methodology allows removal of these behavioral and response demands, making the task a more accurate measure of receptive language ability and arguably a superior outcome measure for clinical trials compared to child-to-clinician assessments.

We found that parents' report of children's knowledge of words and performance on the PVET was related, i.e., when parents reported that the child knew the word (e.g., book) the child looked at the known labeled object, i.e., book, longer compared to the distractor object suggesting that they recognized the item 'book.' This finding is consistent with existing evidence demonstrating that children with ASD spend a longer amount of time looking at known items versus unknown items $\mathrm{s}^{32}$ and lends confidence for using this method as to assess receptive language skills.

For our second aim, we assessed receptive language improvement in children with FXS using the PVET as an outcome measure in a randomized, double-blind, placebo-controlled trial of sertraline. We found that children's looking time to the target object after the object had been labeled was greater in the sertraline group compared to the placebo group suggesting that sertraline may be an effective treatment to improve receptive language in young children with FXS. In contrast, when we used the MSEL and the PLS to examine the effect of sertraline on language improvement, we found no significant improvement in receptive language with these clinical assessments. Although our data suggest that performance of children with FXS on the PVET, the MSEL, and the PLS were related (Figure 4), the PVET was the only measure that was sensitive enough to detect improvements in language comprehension after administration of sertraline in children with FXS. Although our sample size was small, these preliminary findings warrant further examination of eye tracking tasks as an outcome measure for treatment studies with children with FXS given that these tasks are not behaviorally taxing (requiring verbal and motor responses) on the child compared to traditional clinical assessments. Our sample for the placebo-controlled trial of sertraline was a subset of the sample reported in a previous placebo-controlled study by GreissHess et al., ${ }^{18}$ In that study, no significant differences in receptive language improvement in the sertraline or the placebo group were observed when accessed via the MSEL, which was consistent with the current findings. In contrast, a retroactive chart review reported significant improvement in both expressive and receptive language in FXS children treated with sertraline. ${ }^{19}$ When we used the PVET as an outcome measure of language improvement, the PVET could detect these improvements in vocabulary knowledge. Our results suggest that the PVET may be a useful tool to gauge subtle improvements in receptive language that traditional standardized assessments may not reflect. These results demonstrate a need for future studies to replicate our preliminary findings using eye tracking tasks as an outcome measure in treatment studies of children with FXS.

Existing evidence suggests that early development may be an opportune time to implement treatment given the rapid synapse formation and network connectivity observed in the first years of life. ${ }^{43,44}$ In nonhuman primates, developmental changes in serotonin content and binding of serotonin receptors in the brain have been demonstrated. ${ }^{45,46}$ In children with ASD, however, there is a marked disruption in the capacity of serotonin synthesis starting early in life. ${ }^{47}$ Due to this disruption in the first few years of life, not only is it important to deliver potentially beneficial pharmacological SSRI treatments such as sertraline but it is just as critical to detect improvements in domains such as language. The detection of improvements early in life can have cascading effects into the later 
years of life, and our results suggest that the PVET may be a useful tool to detect these subtle yet critical improvements.

Although there was no difference in reported side effects of sertraline compared to the placebo and no serious adverse events in both groups over the course of six months, ${ }^{18}$ long-term follow up visits are critical to better understand the effects of and potential issues with sertraline use in young children. Nonetheless, our study is a novel study using a passive-viewing eye tracking task to assess receptive language improvement in a controlled trial of sertraline in children with FXS and provides evidence for improved language comprehension in young children with FXS with sertraline use. Future studies should not only investigate the effects of long-term sertraline use in young children but also examine the validity of using outcome measures with various methodologies including eye tracking in conjunction with standardized assessments of language skills.

\section{Conclusion}

The results of the current study provide evidence for using eye tracking methodology as a measure of language skill in children with FXS. Using this methodology, we also provide preliminary evidence of sertraline as an effective treatment in improving receptive language in young children with FXS.

\section{Funding}

This project was supported by R01 HD056031 from the NICHD to S. M. Rivera. Other sources of support include the Health Resources and Services Administration (HRSA) of the U.S. Department of Health and Human Services (HHS) under grant number R40MC22641 and T32MH073124 from the National Institute of Mental Health. The information or content and conclusions are those of the authors and should not be construed as the official position or policy of, nor should any endorsements be inferred by HRSA, HHS, or the National Institute of Mental Health or the National Institutes of Health. The project was also supported by the MIND Institute Intellectual and Developmental Disabilities Research Center (U54 HD079125) and the National Fragile X Foundation.

\section{Authors' contributions}

$\mathrm{KY}$ analysed and interpreted the data and was a major contributor in writing the manuscript. JB wrote the method section. SR, KG, and JB designed the eye tracking task. JB and KG collected the data. RH designed the placebo-controlled sertraline study. All authors read and approved the final manuscript.

\section{Acknowledgements}

The authors would like to thank all the families for their time and effort while participating in this study. The authors thank Tawny Bussey for her help in data gathering and double checking all values.

\section{Conflicts of interest}

Author declares there are no conflicts of interest.

\section{References}

1. Brown WT, Hagerman R, Hagerman PJ, et al. The molecular biology of the fragile $\mathrm{X}$ mutation. In: Fragile $\mathrm{X}$ syndrome: Diagnosis, treatment and research. (3rd edn), Johns Hopkins University Press, Maryland, USA. 2002. p.110-135.

2. Crawford DC, Acuña JM, Sherman SL. FMR1 and the fragile $\mathrm{X}$ syndrome: human genome epidemiology review. Genet Med. 2001;3(5):359-371.
3. Abbeduto L, Brady N, Kover S. Language development and fragile $\mathrm{x}$ syndrome: Profiles, syndrome-specificity, and within-syndrome differences. Ment Retard Dev Disabil Res Rev. 2007;13(1):36-46.

4. Berry-Kravis E, Potanos K. Psychopharmacology in fragile X syndrome--Present and future. Ment Retard Dev Disabil Res Rev. 2004;10(1):42-48

5. Budimirovic DB, Kaufmann WE. What can we learn about autism from studying fragile X syndrome. Dev Neurosci. 2011;33(5):379-394.

6. Rousseau F, Heitz D, Biancalana V, et al. Direct diagnosis by DNA analysis of the fragile $\mathrm{x}$ syndrome of mental retardation. $N$ Engl J Med. 1991;325(24):1673-1681.

7. Symons FJ, Byiers BJ, Raspa M, et al. Self-injurious behavior and fragile $\mathrm{X}$ syndrome: Findings from the national fragile $\mathrm{X}$ survey. Am J Intellect Dev Disabil. 2010;115(6):473-481.

8. Yuhas J, Cordeiro L, Tassone F, et al. Brief report: Sensorimotor gating in idiopathic autism and autism associated with fragile $\mathrm{X}$ syndrome. $J$ Autism Dev Disord . 2011;41(2):248-253.

9. Hagerman RJ, Des-Portes V, Gasparini F, et al. Translating molecular advances in Fragile $\mathrm{X}$ syndrome into therapy: A review. J Clin Psychiatry. 2014;75(4):e294-e307.

10. Bagni C, Tassone F, Neri G, et al. Science in medicine Fragile X syndrome: causes, diagnosis, mechanisms, and therapeutics. J Clin Invest. 2012;122(12):4314-4322.

11. Santoro MR, Bray SM, Warren ST. Molecular Mechanisms of Fragile X Syndrome: A Twenty-Year Perspective. Annu Rev Pathol. 2012;7:219-245.

12. Fernández E, Rajan N, Bagni C. The FMRP regulon: From targets to disease convergence. Front Neurosci. 2013;7:191.

13. Schaefer TL, Davenport MH, Erickson CA. Emerging pharmacologic treatment options for fragile X syndrome. Appl Clin Genet. 2015;8:75-93.

14. Chandana SR, Behen ME, Juhász C, et al. Significance of abnormalities in developmental trajectory and asymmetry of cortical serotonin synthesis in autism. Int J Dev Neurosci. 2005;23(2-3):171-182.

15. Hanson AC, Hagerman RJ. Serotonin dysregulation in Fragile $\mathrm{X}$ Syndrome: implications for treatment. Intractable rare Dis Res. 2014;3(4):110-117.

16. Reinhard SM, Razak K, Ethell IM. A delicate balance: role of MMP9 in brain development and pathophysiology of neurodevelopmental disorders. Front Cell Neurosci. 2015;9:280.

17. Meredith RM, De Jong R, Mansvelder HD. Functional rescue of excitatory synaptic transmission in the developing hippocampus in Fmr1-KO mouse. Neurobiol Dis. 2011;41(1):104-110.

18. Greiss-Hess L, Fitzpatrick ES, Nguyen VD, et al. A Randomized Double-Blind, Placebo-Controlled Trial of Sertraline in Young Children with Fragile X Syndrome. J Dev Behav Pediatr. 2016;1-10.

19. Winarni TI, Chonchaiya W, Adams E, et al. Sertraline May Improve Language Developmental Trajectory in Young Children with Fragile X Syndrome: A Retrospective Chart Review. Autism Res Treat. 2012;2012:104317.

20. Steingard RJ, Zimnitzky B, DeMaso DR, et al. Sertraline treatment of transition-associated anxiety and agitation in children with autistic disorder. J Child Adolesc Psychopharmacol. 1997;7(1):9-15.

21. Abbeduto L, Murphy MM, Cawthon SW, et al. Receptive language skills of adolescents and young adults with Down or Fragile X syndrome. Am $J$ Ment Retard. 2003;108(3):149-160.

22. Abbeduto L, Hagerman R. Language and communication in fragile $x$ syndrome. Ment Retard Dev Disabil Res Rev. 1997;3:313-322. 
23. Price J, Roberts J, Vandergrift N, et al. Language comprehension in boys with fragile $\mathrm{X}$ syndrome and boys with Down syndrome. $J$ Intellect Disabil Res. 2007;51:318-326.

24. Kemper MB, Hagerman RJ, Altshul-Stark D. Cognitive profiles of boys with the fragile X syndrome. Am J Med Genet . 1988;30(1-2):191-200.

25. Hooper SR, Hatton D, Sideris J, et al. Executive functions in young males with fragile $\mathrm{X}$ syndrome in comparison to mental age-matched controls: baseline findings from a longitudinal study. Neuropsychology. 2008;22(1):36-47.

26. Cornish K, Sudhalter V, Turk J. Attention and Language in Fragile X. Ment Retard Dev Disabil Res Rev. 2004;10(1):11-16.

27. Baumgardner TL, Reiss AL, Freund LS, et al. Specification of the neurobehavioral phenotype in males with fragile $\mathrm{X}$ syndrome. Pediatrics. 1995;95(5):744-752

28. Wisbeck JM, Huffman LC, Freund L, et al. Cortisol and social stressors in children with fragile X: a pilot study. [Internet]. Journal of developmental and behavioral pediatrics. 2000;278-282.

29. Lesniak-Karpiak K, Mazzocco MM, Ross JL. Behavioral assessment of social anxiety in females with Turner or fragile X syndrome. J Autism Dev Disord. 2003;33(1):55-67.

30. Cordeiro L, Ballinger E, Hagerman R, et al. Clinical assessment of DSM-IV anxiety disorders in fragile $\mathrm{X}$ syndrome: prevalence and characterization. J Neurodev Disord. 2011;3(1):57-67.

31. Fernald A, Zangl R, Portillo A, et al. Looking while listening: Using eye movements to monitor spoken language comprehension by infants and young children. Developmental Psycholinguistics: On-line methods in children's langugage processing. John Benjamins, Netherlands. 2008. p.97-135.

32. Brady N, Anderson C, Hahn L, et al. Eye Tracking as a Measure of Receptive Vocabulary in Children with Austim Spectrum Disorders. Augment Altern Commun. 2014;30(2):147-159.

33. EM .Mullen scales of early learning. AGS Editio. Circle Pines, MN American Guidance Service, USA. 1995.

34. Zimmerman I, Steiner V, Pond R. Preschool Language Scale, Fourth Edition. San Antonio, TX: The Psychological Corporation. 2002.

35. Fenson L, Dale PS, Reznick JS, et al. The MacArthur Communicative Development Inventories: User's guide and technical manual. Singular Publishing Group, California, USA. 1993. p.1-208.
36. Snodgrass JG, Vanderwert M. A standardized set of 260 pictures: Norms for name agreement, image agreement, familiarity, and visual complexity. J Exp Psychol Hum Learn Mem. 1980;6(2):174-215.

37. Rossion B, Pourtois G. Revisiting snodgrass and Vanderwert's object database: Color and texture improve object recognition. J Vis. 2001;1:413.

38. Finestack, Lizbeth H, Richmond, et al. Language Development in Individuals with Fragile $\mathrm{X}$ Syndrome. Top Lang Disord. 2009;29(2):133-148.

39. Barry-Anwar RA, Burris JL, Graf Estes K, et al. Caregivers and strangers: The influence of familiarity on gaze following and learning. Infant Behav Dev. 2017;46:46-58.

40. Volden J, Smith IM, Szatmari P, et al. Using the Preschool Language Scale, Fourth Edition to Characterize Language in Preschoolers With Autism Spectrum Disorders. Am J Speech-Language Pathol 2011;20(3):200-208.

41. Zingerevich C, Greiss-Hess L, Lemons-Chitwood K, et al. Motor abilities of children diagnosed with fragile $\mathrm{X}$ syndrome with and without autism. J Intellect Disabil Res. 2009;53(1):11-18.

42. Reiss AL, Hall SS. Fragile X Syndrome: Assessment and Treatment Implications. Child Adolesc Psychiatr Clin N Am. 2007;16(3):663-675.

43. Lo ST, Festen DAM, Tummers-de Lind van Wijngaarden RFA, et al. Beneficial Effects of Long-Term Growth Hormone Treatment on Adaptive Functioning in Infants With Prader-Willi Syndrome. Am J Intellect Dev Disabil. 2015;120(4):315-327.

44. Webb SJ, Jones EJ, Kelly J, et al. The motivation for very early intervention for infants at high risk for autism spectrum disorders. Int $J$ Speech Lang Pathol. 2015;16(1):36-42.

45. Goldman-Rakic PS, Brown RM. Postnatal development of monoamine content and synthesis in the cerebral cortex of rhesus monkeys. Brain Res. 1982;256(3):339-349.

46. Lidow MS, Goldman Rakic PS, Rakic P. Synchronized overproduction of neurotransmitter receptors in diverse regions of the primate cerebral cortex. Proc Natl Acad Sci USA. 1991;88(22):10218-10221.

47. Chugani DC, Muzik O, Behen M, et al. Developmental changes in brain serotonin synthesis capacity in autistic and nonautistic children. Ann Neurol. 1999;45(3):287-295. 